\title{
Preparation \& Characterization of Electrically Conducting Starch-Gelatin Blended Films Doped With Polyaniline / Graphene Oxide
}

\author{
U. Venkateswarlu \\ Senior Scientist, Advanced Materials Lab, Central Leather Research Institute, \\ Adyar, Chennai-600020, India \\ Email: venkateswaralu@clri.res.in, vummadisetty@gmail.com
}

\begin{abstract}
In the present study a conducting biopolymer composite made of gelatin-starch-polyaniline-graphene was synthesized \& characterized which can be used as a base material in fabrication of transducers. Initially starch and gelatin blended films were optimized by Tensile strength and Electrical conductivity. Later graphene oxide and Polyaniline (PANI) were doped on to Starch-Gelatin blended films and these films were also characterized by using FTIR and Electrical conductivity. It was concluded that the composite that contains 2:1 ratio of Starch and Gelatin blend is optimized with respect to good tensile strength and excellent electrical conductivity. The $20 \mathrm{mg} / \mathrm{ml}$ of PANI doping on to Starch-Gelatin blended films and $1 \mathrm{mg} / \mathrm{ml}$ of graphene oxide doping on to Starch-Gelatin blended films were optimized and they showed excellent conductivity of $5.8 \times 10^{-9} \mathrm{~S} / \mathrm{m}$ and $7.6 \times 10^{-9} \mathrm{~S} / \mathrm{m}$ respectively.

Keywords: Starch, gelatin, polyaniline, graphene, FTIR, Tensile strength, Electrical conductivity.
\end{abstract}

\section{INTRODUCTION}

Gelatin is a translucent, colorless, brittle (when dry), flavorless food ingredient that is derived from collagen obtained from various animal body parts. The gelatin film is electrically insulator when it is in dry condition but when it is humidified with heavy water vapor it conducts electricity this is due to protons were replaced by deuterium nuclei in gelatin film as found by Niven [1]. Giovanni Landi [2] synthesized gelatin/graphene composite and analyzed by means of current density-voltage and the electrical impedance measurements. He found that graphene sheets to the biopolymer results in an enhancement of the electrical power transfer to the load due to a decreasing series resistance, without a decrease of the cell open circuit voltage. Electrical conductivity of starch in water is studied by Morales [3] and found that the electrical conductivity of the water-starch depends on moisture and temperature. In wet condition electrical conductivity increases due to carbonyl group and in dry condition the ions are seized and conduct no current. Marcotte [4] has studied about electrical conductivity of gelatin-starch composite at $25^{\circ} \mathrm{C}$ and at $100^{\circ} \mathrm{C}$ and found that at lower temperature it shows a minimal electrical conductivity than at higher temperature. Serge Ostrovidov [5] has studied about composite gelatinpolyaniline (PANI) Nanofibers doped with camphor sulfonic acid (CSA) fabricated by electro spinning and used as a substrates to culture $\mathrm{C} 2 \mathrm{C} 12$ myoblast cells. This composite material scaffolds combining topographical and electrically conductive cues may be useful to direct skeletal muscle cell organization and to improve cellular maturation, functionality, and tissue formation. Ehsan Nazarzadeh zareh [6] has prepared biodegradable conductive blends based on polyaniline/starch in acidic medium. Various polyaniline/starch ratios $(\mathrm{w} / \mathrm{w} \%)$ were prepared to study the structure of the final blends. The conductivity of blends was measured and it was found that the electrical conductivity of the blends was increased by increasing the amount of polyaniline (PANI). Priscilla [7] have prepared Bio-Nano composite films by casting an aqueous suspension of acetylated starch (ST) and poly(vinyl alcohol) (PVA) loaded with graphene oxide (GO). The Nano composites have displayed improved thermal and electrical properties when the amount of the GO phase is increased and properly converted to RGO. The thermal diffusivity and electrical resistivity of STGO Nano composites have increased one order and decreased two orders of magnitude, respectively, after the photochemical treatment. Since the combination of gelatin, starch, polyaniline and graphene is not available in prior art, we proposed to prepare and optimize these combination of biopolymer and characterized the films using FTIR, Tensile strength and dc/ac conductivity.

\section{MATERIALS \& METHODS 2.1 Materials}




\section{International Journal of Research in Advent Technology, Vol.7, No.1, January 2019 E-ISSN: 2321-9637 \\ Available online at www.ijrat.org}

Gelatin was purchased from HIMEDIA, Mumbai, India and used as such without further purification. Potato starch was purchased from SIGMA-ALDRICH, India and used as such without purification. Other materials used in our study are of Laboratory Analytical grade.

\subsection{Methods}

\subsubsection{Synthesis of polyvinyl alcohol (PANI)}

$100 \mathrm{ml}$ of aniline is mixed with $900 \mathrm{ml}$ of $2.4 \mathrm{M}$ hydrochloric acid in a $1000 \mathrm{ml}$ glass beaker. It is placed in an ice bath and $100 \mathrm{ml}$ of $0.44 \mathrm{M}$ solution of potassium dichromate was added drop wise into the solution. The mixer is stirred before and after addition of potassium dichromate. The setup was left as such for an hour for reaction to take place. The reaction mixer was then filtered and dried in a hot air oven. The dried precipitate was subjected to acetone and water wash. Washed powder is again dried in hot air oven to obtain Emeraldine salt in green color.

2.2.2 Graphene Oxide Synthesis (GO)

Graphene Oxide was synthesized according to Hummers method [8].

2.2.3 Thermal reduction of Graphene oxide to Graphene (GRA)

Graphene oxide has to be reduced thermally to increase the efficiency of doping with polymer blend. In this regard we synthesized the Graphene in our laboratory. $20 \mathrm{mg}$ of the so prepared graphene oxide were dispersed in to $6 \mathrm{ml}$ water under mild ultrasound. Then $30 \mathrm{ml}$ of
Dimethyl Acetamide (DMAc) was added and sonificated for few minutes to obtain a transparent yellow-brown suspension. It is then placed in a microwave oven under nitrogen gas at 800 Watt for 10 minutes. The solution is filtered and treated with $30 \mathrm{ml}$ of ethanol for three times and later with $15 \mathrm{ml}$ of ultrapure water under mild ultrasound. After that the mixture is dried at $80^{\circ} \mathrm{C}$ for 24 hours to get black powder of graphene. $10 \mathrm{mg}$ of obtained graphene was dispersed in $10 \mathrm{ml}$ of DMAc under mild ultrasound to obtain graphene suspension for doping into optimized gelatinstarch composite.

\subsubsection{Preparation of Gelatin Solution}

$5 \mathrm{gm}$ of gelatin powder is dissolved into $100 \mathrm{ml}$ of distilled water and heated the solution to $90^{\circ} \mathrm{C}$ for about 30 minutes to get homogeneous solution. It is then cooled in room temperature and stored for further use.

2.2.5. Preparation of Starch Solution

$1 \mathrm{gm}$ of starch powder is added to $100 \mathrm{ml}$ of distilled water in a $500 \mathrm{ml}$ glass beaker and heated to $90^{\circ} \mathrm{C}$ for about 30 minutes until to get homogeneous solution. It is then cooled in room temperature and stored for further use.

2.2.6. Preparation of Starch-Gelatin blend

Above prepared Gelatin and Starch solutions were added at different proportion as per below table. 1 to get six samples for optimization.

Table.1. Gelatin-Starch blends

\begin{tabular}{|l|l|l|l|}
\hline $\begin{array}{l}\text { Concentration Ratio } \\
\text { (STA:GEL) }\end{array}$ & Sample-ID & Starch solution $(\mathrm{ml})$ & Gelatin solution (ml) \\
\hline $10: 1$ & $\mathrm{C} 1$ & 18.2 & 1.8 \\
\hline $5: 1$ & $\mathrm{C} 2$ & 16.7 & 3.3 \\
\hline $2: 1$ & $\mathrm{C} 3$ & 13.3 & 6.7 \\
\hline $1: 1$ & $\mathrm{C} 4$ & 10 & 10 \\
\hline $1: 2$ & $\mathrm{C} 5$ & 6.7 & 13.3 \\
\hline $1: 5$ & C6 & 3.3 & 16.7 \\
\hline
\end{tabular}

These six samples were placed in glass petri dish plates separately and kept them in an over at $40^{\circ} \mathrm{C}$ for 24 hours to get films. These films are used for further analysis.

\subsubsection{Resistivity}

Electrical DC resistivity of the Gelatin-Starch films was determined by using "Mastech MAS830L Digital Pocket Multimeter".

\subsubsection{AC Conductivity}

Electrical ac conductivity of the films of GelatinStarch was determined by using "Mastech MAS830L Digital Pocket Multimeter" and SCIENTIFIC $5 \mathrm{MHz}$ Function Generator SM5072. The equivalent electrical circuit to measure ac conductivity of the films is given in below fig.1. Sample is placed between copper plates to give good electrical contact to the films. 


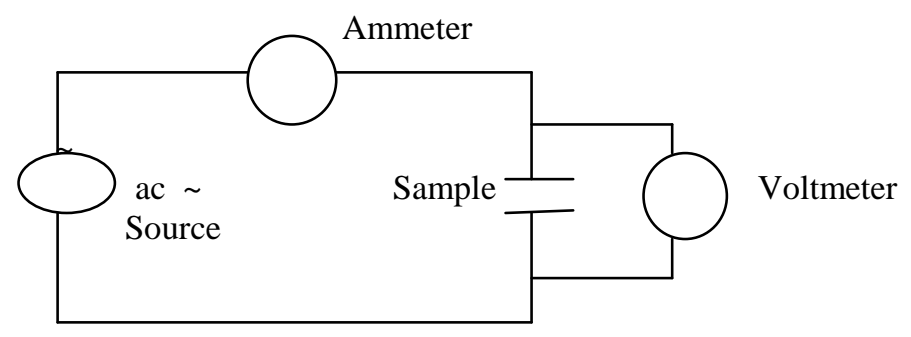

Fig.1. Circuit diagram to measure ac Conductivity

\subsubsection{FTIR Analysis}

FTIR spectroscopy for samples is analyzed using Nicolet 170 SX, Thermo Fisher Scientific Inc., USA in the range of $400-4000 \mathrm{~cm}-1$. One $\mathrm{mg}$ of dry sample was mixed with $100 \mathrm{mg}$ of dry $\mathrm{KBr}$ and the mixture is pressed in to a disk for spectral analysis.

\subsubsection{Tensile strength}

The samples of Gelatin-Starch were characterized for their Tensile strength using INSTRON 1405 at a speed of $5 \mathrm{~mm} / \mathrm{min}$. Two dumbbell shaped specimens of $5 \mathrm{~mm}$ wide and $10 \mathrm{~mm}$ length were punched out using a die for each films. Mechanical properties such as Tensile strength (MPa) and elongation at break (\%) were measured.

\section{RESULTS \& DISCUSSION}

3.1 Tensile strength of Starch-Gelatin Blended films The Tensile strength test was conducted on different blend ratio of Starch-Gelatin films (C1to C6) and the results are plotted in below fig. 2 .

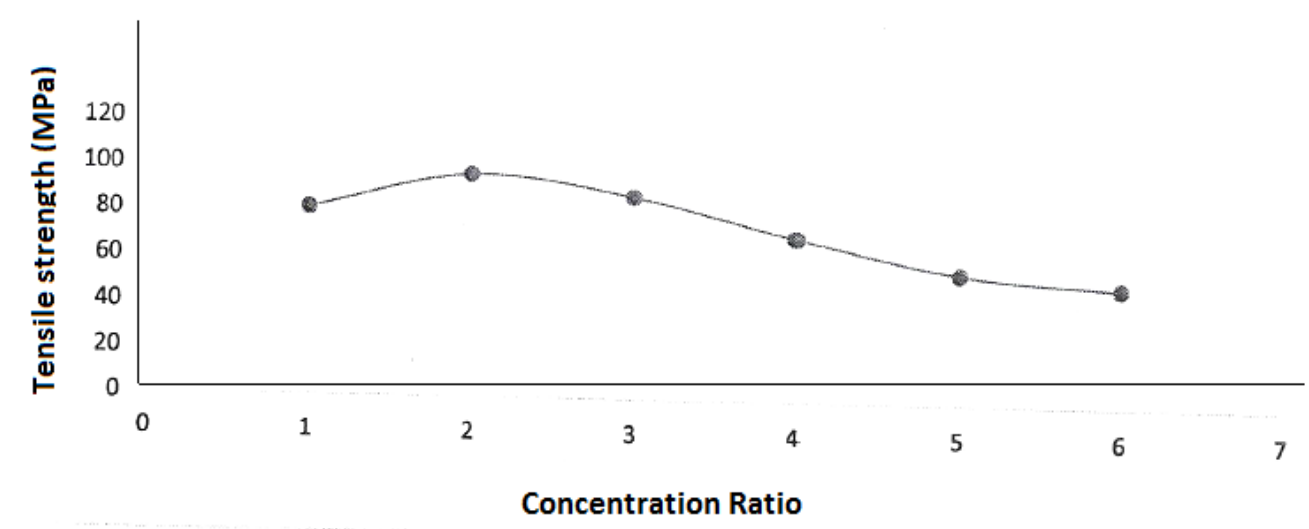

Fig.2. Tensile strength of Starch-Gelatin blended films

The tensile strength increases with increase in starch concentration initially and reaches a maximum at 2:1 ratio of starch and gelatin and then decreases with increase in starch ratio. This is due to the fact that carbohydrates in starch get stabilized by means of glycoside linkage and gelatin by means of hydrophilic interactions. At maximum peak, all the available carbohydrates were linked to gelatin and after that unreacted carbohydrate gives lesser tensile strength.

\subsection{Resistance of Starch-Gelatin blended films}

The DC electrical resistance of the Starch-Gelatin blended films at different blend ratio from $\mathrm{C} 1$ to $\mathrm{C} 6$ is shown in below fig. 3 . 


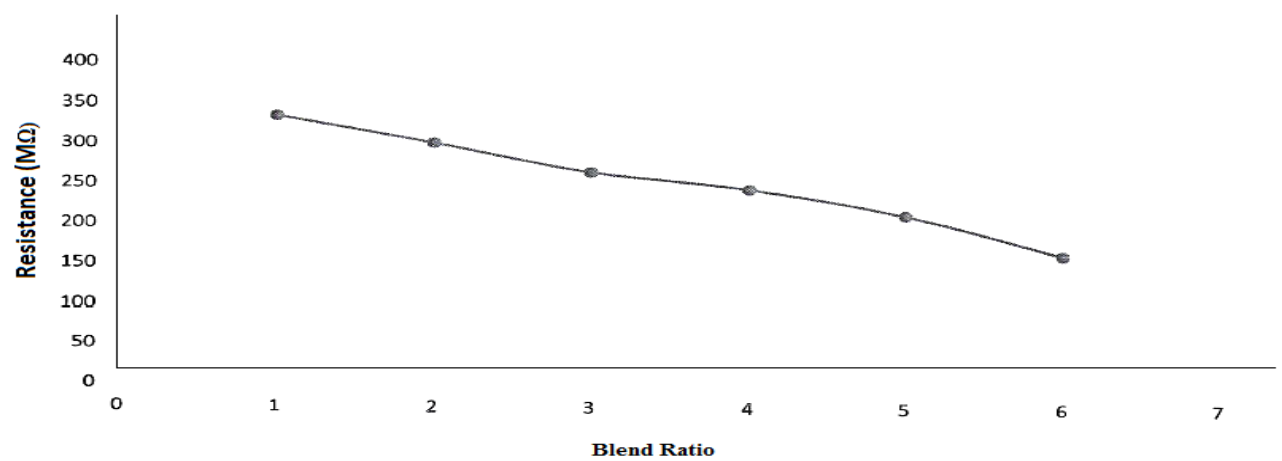

Fig.3. DC Resistance of Starch-Gelatin blended films

The dc resistance decreases with increase in starch concentration that means dc conductivity increases with starch ratio. This is due to availability of more carbohydrates in the films which contributes to electrical conductivity.

\subsection{AC Conductivity of Starch-Gelatin blended} Films

The electrical ac conductivity of the films of different blend ratio from samples $\mathrm{C} 1$ to $\mathrm{C} 6$ interms of current verses ac frequency is shown in below fig. 4 .

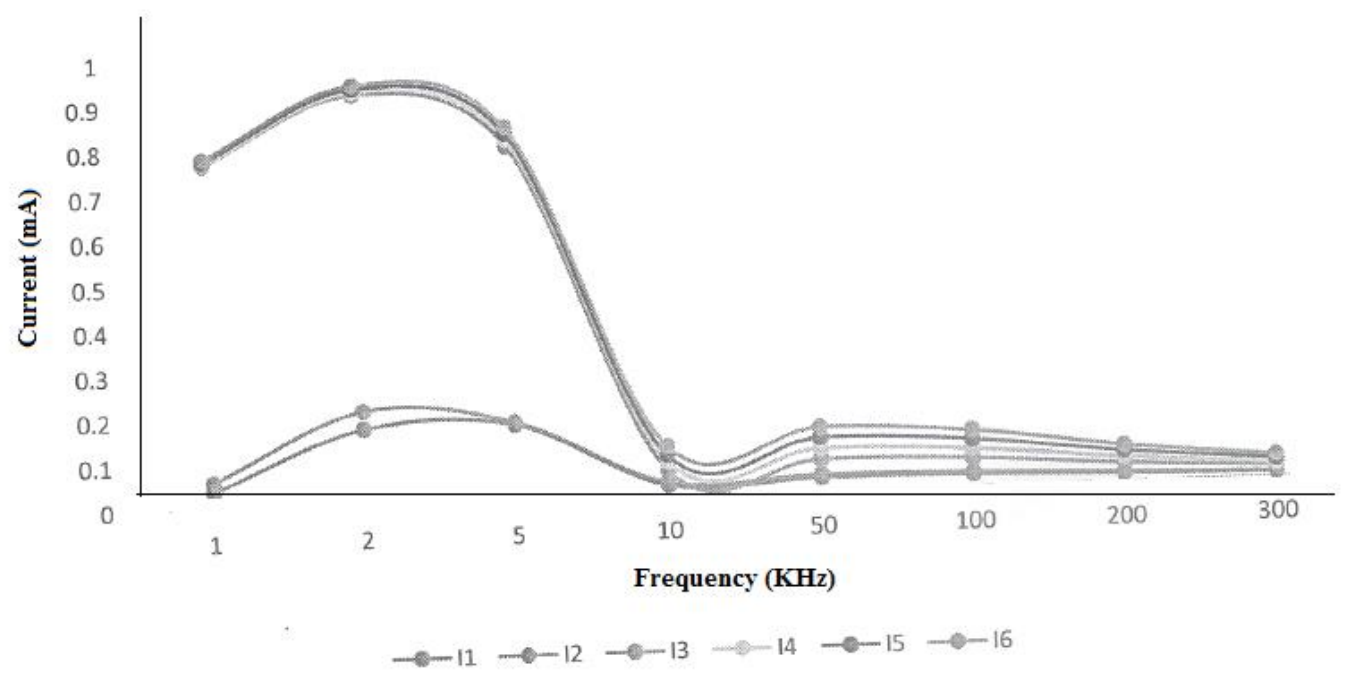

Fig.4. AC conductivity of gelatin-starch films

The ac current through the samples $\mathrm{C} 1$ to $\mathrm{C} 2$ shows maximum at around $2 \mathrm{kHz}$ frequency and then decreases to a minimum at around $10 \mathrm{kHz}$ and after that almost same amount of current flows through all the samples. First time I found that there is an ac frequency after which a constant minimum current flows through all sample even though by increasing the ac frequency and I called this frequency as a "Threshold frequency". This threshold frequency is characteristic to the composition of the conducting biopolymer. In this case the threshold frequency for gelatin-starch biopolymer is $10 \mathrm{kHz}$. From the figure, it was also found that the ac current through the sample increases from $0 \mathrm{kHz}$ and reaches maximum at $2 \mathrm{kHz}$ and then decreases to threshold frequency. This phenomenon is same for all samples (C1-C6). For samples $1 \& 2$ almost same amount of low current flows but for samples from C3-C6 almost same amount of high current flows. Low amount of current values is due to availability of less number of carbohydrates and high value of current is due to availability of more carbohydrates in the samples which contributes to electron flow. It was found that there exist a frequency band during which more current flows through the sample and I called this frequency band as a "Bandwidth". This bandwidth is also characteristic to the composition of the conducting biopolymer. 


\subsection{FTIR Analysis of Graphene oxide doped Starch- Gelatin blended films}

FTIR spectra of graphene oxide doped on to StarchGelatin blended film and reduced graphene oxide doped on to Starch-Gelatin blended film is shown in fig.5.

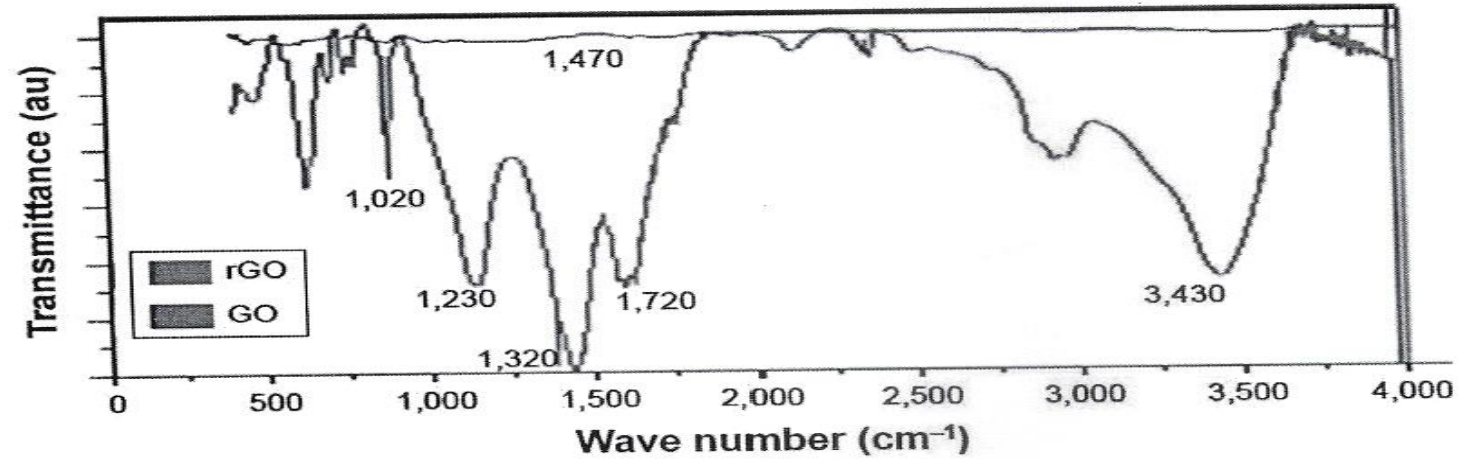

Fig.5. FTIR Analysis of Graphene oxide doped Starch-Gelatin blended films

The FTIR spectrum of reduced graphene oxide blend film shows a flat curve. This means that graphene substance dominate in the film. The spectrum of graphene oxide blend film shows peak at $1020 \mathrm{~cm}^{-1}$ corresponds to stretching vibration of $\mathrm{C}-\mathrm{O}-\mathrm{C}$ bonds of alkyl groups, peak at $1230 \mathrm{~cm}^{-1}$ is due to $\mathrm{C}-\mathrm{OH}$ bond, peak at $1320 \mathrm{~cm}^{-1}$ and $3430 \mathrm{~cm}^{-1}$ is due to other oxygenated functional groups includes $\mathrm{OH}$ group and peak at $1720 \mathrm{~cm}^{-1}$ is due to the contribution of $\mathrm{C}=\mathrm{O}$ bonds in carboxylic group. This FTIR spectrum confirms the phenomenon of cross linking of graphene oxide on to Starch-Gelatin blended films. 3.5 DC Resistivity of Doped and un-doped films The electrical dc resistivity of un-doped StarchGelatin blended film (D0), polyaniline doped on to Starch-Gelatin blended film (D1) and graphene doped on to Starch-Gelatin blended film (D2) are shown in fig.6.

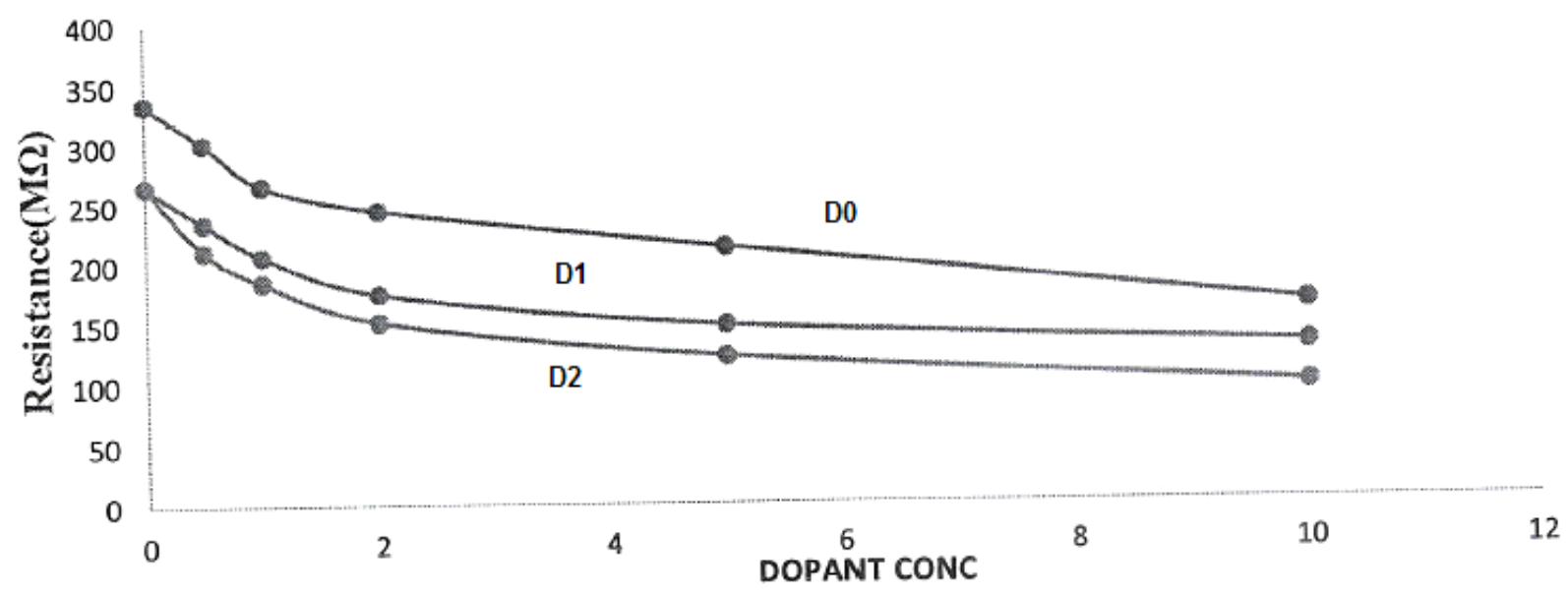

Fig.6. DC Resistivity of D0-D1-D2 composite films

The doping concentration of PANI onto the StarchGelatin blended film is $20 \mathrm{mg} / \mathrm{ml}$ whereas the doping concentration of graphene oxide on to Starch-Gelatin blended film is $1 \mathrm{mg} / \mathrm{ml}$. The dc resistance of undoped composite (D0) is higher than that of PANI doped Starch-Gelatin blended film and also that of Graphene Oxide doped Starch-Gelatin blended film.
Even though the concentration of PANI is 20 fold higher than that of graphene oxide, the resistance is higher than that of graphene oxide.

3.6. AC Conductivity of PANI doped Starch-Gelatin blended film 
The ac conductivity of different doping concentration of PANI (D0 $\rightarrow$ D5) on to Starch-Gelatin blended

films is shown in below figure 7 .

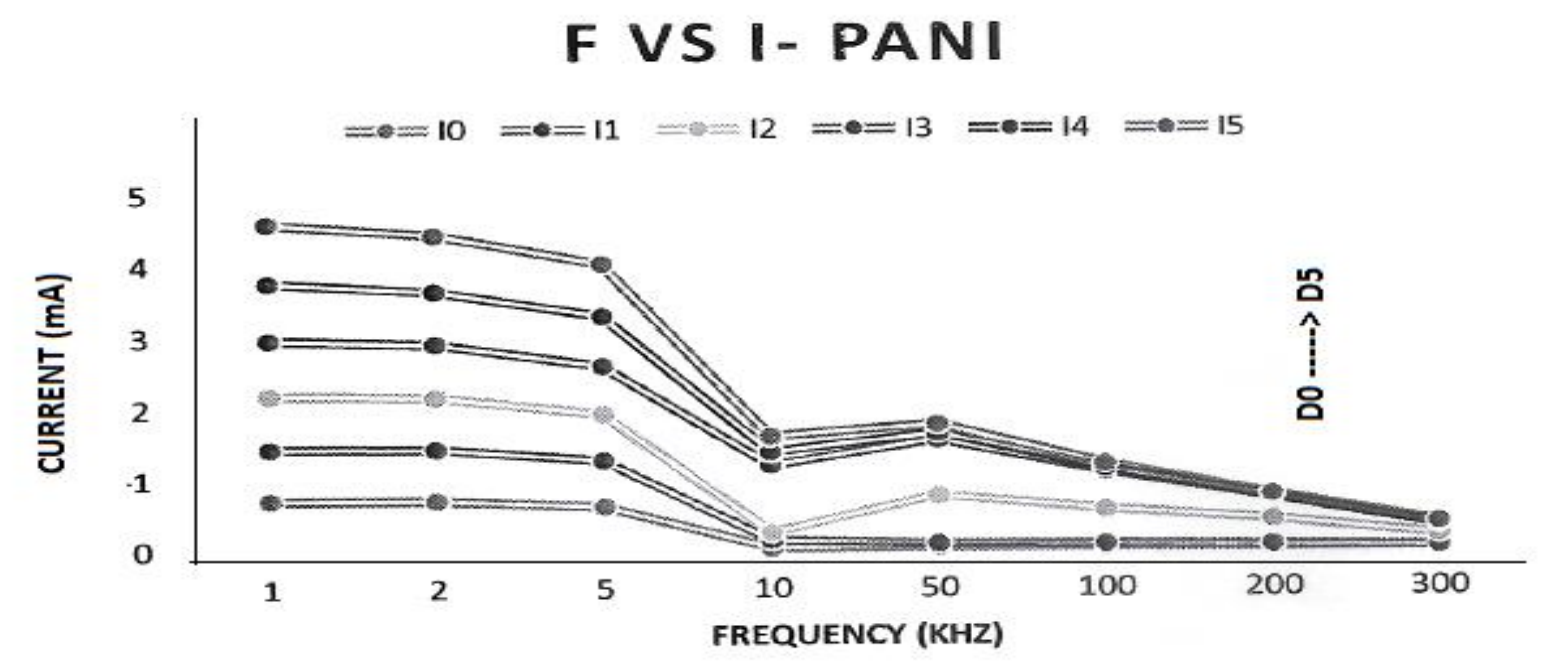

Fig.7. ac current verses ac frequency of PANI doped Starch-Gelatin blended film

From the plot it is evident that the current flowing through the un-doped sample (D0) shows less than that of D1 to D5. If doping concentration of PANI on to Starch-Gelatin blended film (D1 $\rightarrow$ D5) increases the current flow is also increases, this is due to availability of more number of charge carriers in PANI concentration. In this case the threshold frequency was found to be $10 \mathrm{kHz}$ for samples D0 $\rightarrow$ D2 and $300 \mathrm{kHz}$ for samples D3 $\rightarrow$ D5.

3.7. AC Conductivity of Graphene oxide doped Starch-Gelatin blended films

The ac conductivity of different doping concentration of Graphene oxide (D0 $\rightarrow$ D5) on to Starch-Gelatin blended films is shown in below figure 8 .

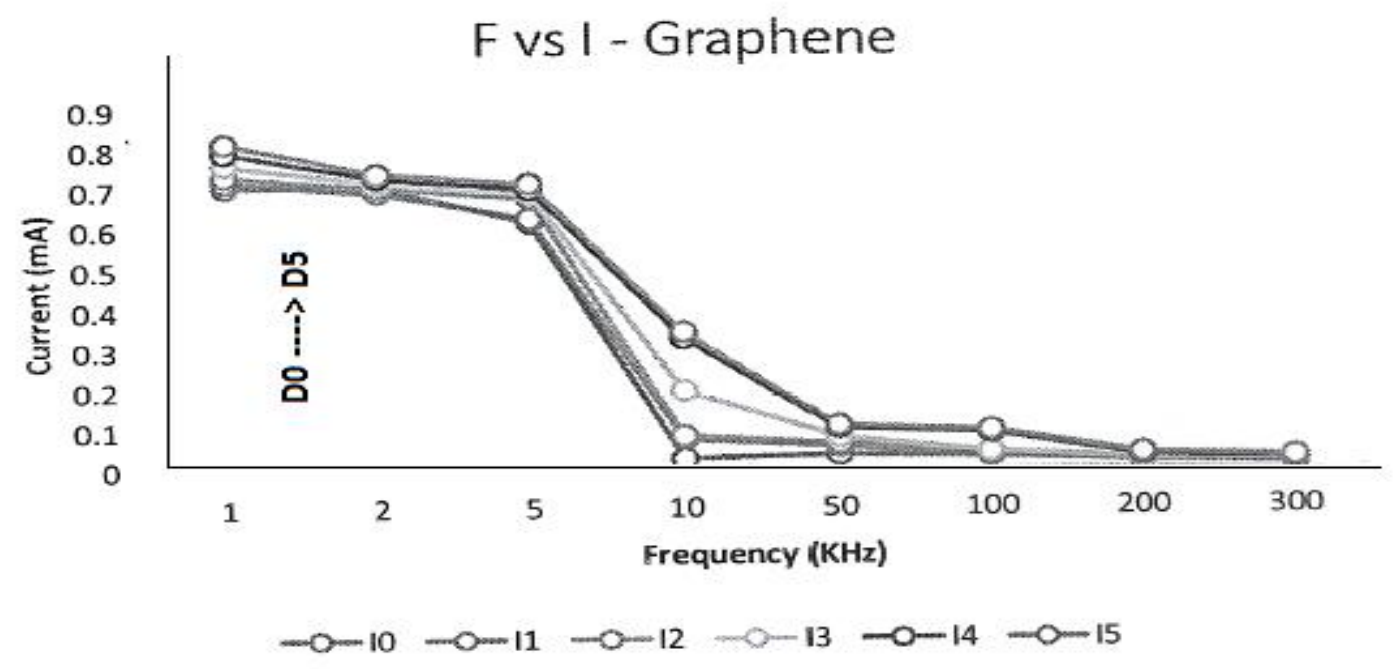

Fig.8. ac current verses ac frequency of Graphene oxide doped Starch-Gelatin blended films

From above figure it is evident that the current flowing through the un-doped sample (D0) shows less than that of D1 to D5. If doping concentration of Graphene oxide (D1 $\rightarrow$ D5) increases then current flow also increases very slightly. This is due to availability of number of charge carriers in graphene oxide concentration. In this case the threshold frequency was found to be $10 \mathrm{kHz}$ for samples D0 $\rightarrow \mathrm{D} 2$ and $50 \mathrm{kHz}$ for samples D3 $\rightarrow$ D5. 


\section{International Journal of Research in Advent Technology, Vol.7, No.1, January 2019 E-ISSN: 2321-9637 \\ Available online at www.ijrat.org}

\section{CONCLUSION}

The films with 2:1 ratios of Starch-Gelatin blended film were found to be optimum interms of tensile strength and electrical conductivity. The hydrophilic groups present in starch readily get ionized and conducts electricity in Starch-Gelatin blended films. The PANI doped optimized sample has conductivity of $5.8 \times 10^{-9} \mathrm{~S} / \mathrm{m}$ and Graphene oxide doped optimized sample has conductivity of $7.6 \times 10^{-9} \mathrm{~S} / \mathrm{m}$. These conductivity values are in close relation to semi-conducting materials and hence one can use these materials in semiconductor industry.

\section{REFERENCES}

[1] C. D. Niven. "The Electrical Conductivity of Gelatin film humidified with Heavy Water Vapor". Canadian Journal of Physics, volume 39(5), pages 657-661, 1961.

[2] Giovanni Landi, Andrea Sorrentino, Salvatore Iannace and Heinrich C. Neitzert. "Electrical Characterization and Modeling of a Gelatin/Graphene System". Advances in Condensed Matter Physics, pages 1-5, 2015.

[3] Pedro A Vazquez-Landaverde, Marcela Gaytan-Martinez, Jorge A Huerta-Ruelas and Eduardo Morales-Sanchez. "Electrical Conductivity of Heated Cornstarch-Water mixtures". Journal of Food processing Engineering, volume 32, pages 817-827, 2009.

[4] M. Marcotte, J.P.G. Piette and H.S. Ramaswamy. "Electrical Conductivities of Hydrocolloid Solutions". Journal of Food processing Engineering, volume 21, pages 503-520, 2007.

[5] Serge Ostrovidov, Majid Ebrahimi, Hojae Bae, Hung Kim Nguyen, Sahar Salehi, Sang Bok Kim, Akichika Kumatani, Tomokazu Matsue, Xuetao Shi, Ken Nakajima, Shizu Hidema, Makoto Osanai and Ali Khademhosseini. "Gelatin-Polyaniline Composite Nanofibers Enhanced Excitation-Contraction Coupling System Maturation in Myotubes". Applied Materials \& Interfaces, volume 9, pages 4244442458, 2017.

[6] Ehsan Nazarzadeh zareh, Peyman Najafi Moghadam, Elham Azariyan and Iman Sharifian. "Conductive and Biodegradable Polyaniline/Starch Blends and Their Composites with Polystyrene". Iranian Polymer Journal, volume 20, pages 319-328, 2011.

[7] Priscilla P.Peregrino,Maria J.A.Sales, Mauro F.P.da Silva, Maria A.G.Soler, Luiz F.L.da Silva, Sanclayton G.C.Moreira and Leonardo
G.Paterno. "Thermal and electrical properties of starch-graphene oxide Nano composites improved by photochemical treatment". Carbohydrate Polymers, volume 106, pages 305311, 2014.

[8] William S. Hummers Jr and Richard E. Offeman. "Preparation of Graphitic Oxide". Journal of the American Chemical Society, volume 80, pages 1339-1339, 1958. 